\title{
Multiple Positive Solutions of a Singular Semipositone Integral Boundary Value Problem for Fractional $q$-Derivatives Equation
}

\author{
Yulin Zhao, ${ }^{1}$ Guobing Ye, ${ }^{1}$ and Haibo Chen $^{2}$ \\ ${ }^{1}$ School of Science, Hunan University of Technology, Zhuzhou, Hunan 412007, China \\ ${ }^{2}$ Department of Mathematics, Central South University, Changsha, Hunan 410075, China \\ Correspondence should be addressed to Yulin Zhao; zhaoylch@sina.com
}

Received 24 August 2012; Revised 7 December 2012; Accepted 11 January 2013

Academic Editor: Yong Hong Wu

Copyright (C) 2013 Yulin Zhao et al. This is an open access article distributed under the Creative Commons Attribution License, which permits unrestricted use, distribution, and reproduction in any medium, provided the original work is properly cited.

By using the fixed point index theorem, this paper investigates a class of singular semipositone integral boundary value problem for fractional $q$-derivatives equations and obtains sufficient conditions for the existence of at least two and at least three positive solutions. Further, an example is given to illustrate the applications of our main results.

\section{Introduction}

Studies on $q$-difference equations appeared already at the beginning of the 20th century in intensive works especially by Jackson [1], Carmichael [2], and other authors such as Poincare, Picard and, Ramanujan [3]. Up to date, it has evolved into a multidisciplinary subject, for example, see [47] and the references therein. For some recent work on $q$ difference equations, we refer the reader to the papers [8$21]$, and the basic definitions and properties of $q$-difference calculus can be found in the book $[3,22]$. On the other hand, fractional differential equations have gained importance due to their numerous applications in many fields of science and engineering including fluid flow, rheology, diffusive transport akin to diffusion, electrical networks, and probability [23]. Many researchers studied the existence of solutions to fractional boundary value problems, for example, [24-35] and the references therein.

The fractional $q$-difference calculus had its origin in the works by Al-Salam [36] and Agarwal [37]. More recently, perhaps due to the explosion in research within the fractional differential calculus setting, new developments in this theory of fractional $q$-difference calculus were made, specifically, $q$ analogues of the integral and differential fractional operators properties such as the Mittag-Leffler function, the $q$-Laplace transform, and $q$-Taylor's formula $[3,13,22,38]$, just to mention some.
However, the theory of boundary value problems for nonlinear $q$-difference equations is still in the initial stage and many aspects of this theory need to be explored.

In [17], Ferreira considered a Dirichlet type nonlinear qdifference boundary value problem as follows:

$$
\begin{gathered}
D_{q}^{\alpha} u(t)+f(u(t))=0, \quad 0<t<1,1<\alpha \leq 2, \\
u(0)=u(1)=0 .
\end{gathered}
$$

By applying a fixed point theorem in cones, sufficient conditions for the existence of nontrivial solutions were enunciated.

In other paper, Ferreira [18] studied the existence of positive solutions to nonlinear $q$-difference boundary value problem as follows:

$$
\begin{gathered}
D_{q}^{\alpha} u(t)+f(u(t))=0, \quad 0<t<1,2<\alpha \leq 3, \\
u(0)=D_{q} u(0)=0, \quad D_{q} u(1)=\beta \geq 0 .
\end{gathered}
$$

By using a fixed point theorem in a cone, El-Shahed and Al-Askar [19] were concerned with the existence of positive solutions to nonlinear $q$-difference equation:

$$
\begin{aligned}
& { }_{C} D_{q}^{\alpha} u(t)+a(t) f(u(t))=0, \quad 0<t \leq 1,2<\alpha \leq 3, \\
& u(0)=D_{q}^{2} u(0)=0, \quad a D_{q} u(1)+b D_{q}^{2} u(1)=0,
\end{aligned}
$$


where $a, b \geq 0$ and ${ }_{C} D_{q}^{\alpha}$ is the fractional $q$-derivatives of the Caputo type.

Recently, Liang and Zhang [20] discussed the following nonlinear $q$-fractional three-point boundary value problem:

$$
\begin{gathered}
\left(D_{q}^{\alpha} u\right)(t)+f(t, u(t))=0, \quad 0<t<1,2<\alpha \leq 3, \\
u(0)=\left(D_{q} u\right)(0)=0, \quad D_{q} u(1)=\beta D_{q} u(\eta) .
\end{gathered}
$$

By using a fixed point theorem in partially ordered sets, the authors obtained sufficient conditions for the existence and uniqueness of positive and nondecreasing solutions to the above boundary value problem.

In [21], Graef and Kong investigated the following boundary value problem with fractional $q$-derivatives:

$$
\begin{gathered}
\left(D_{q}^{\alpha} u\right)(t)+f(t, u(t))=0, \quad 0<t<1, \\
n-1<\alpha \leq n, n \in \mathbf{N}, \\
\left(D_{q}^{i} u\right)(0)=0, \quad i=0, \ldots, n-2, \\
b D_{q} u(1)=\sum_{j=1}^{m} a_{j} D_{q} u\left(t_{j}\right)+\lambda,
\end{gathered}
$$

where $\lambda \geq 0$ is a parameter, and the uniqueness, existence, and nonexistence of positive solutions are considered in terms of different ranges of $\lambda$.

Furthermore, Ahmad et al. [11] studied the following nonlinear fractional $q$-difference equation with nonlocal boundary conditions

$$
\begin{gathered}
\left({ }_{C} D_{q}^{\alpha} u\right)(t)=f(t, u(t)), \quad 0 \leq t \leq 1,1<\alpha \leq 2, \\
a_{1} u(0)-b_{1} D_{q} u(0)=c_{1} u\left(\eta_{1}\right), \\
a_{2} u(1)+b_{2} D_{q} u(1)=c_{2} u\left(\eta_{2}\right),
\end{gathered}
$$

where ${ }_{C} D_{q}^{\alpha}$ is the fractional $q$-derivative of the Caputo type, and $a_{i}, b_{i}, c_{i}, \eta_{i} \in \mathbf{R}$. The existence of solutions for the problem is shown by applying some well-known tools of fixed point theory such as Banach's contraction principle, Krasnoselskii's fixed point theorem, and the Leray-Schauder nonlinear alternative.

It is known that the fractional q-derivative of RiemannLiouville type played an important role in the development of the theory of fractional $q$-derivatives and $q$-integrals and for its applications in pure mathematics, and is a useful tool in the description of nonconservative models. The details can be found in [22].

Since $q$-calculus has a tremendous potential for applications $[3,22]$, we find it pertinent to investigate problems in this field. Motivated by the papers [20, 21, 30], we will deal with the following integral boundary value problem of nonlinear fractional $q$-derivatives equation:

$$
\left(D_{q}^{\alpha} u\right)(t)+f(t, u(t))=0, \quad t \in(0,1), 2<\alpha \leq 3,
$$

subject to the boundary conditions

$$
u(0)=\left(D_{q} u\right)(0)=0, \quad u(1)=\mu \int_{0}^{1} u(s) d_{q} s,
$$

where $q \in(0,1), \mu$ is parameter with $0<\mu<[\alpha]_{q}$, $D_{q}^{\alpha}$ is the $q$-derivative of Riemann-Liouville type of order $\alpha, f:[0,1] \times \mathbf{R}_{0}^{+} \rightarrow \mathbf{R}$ is continuous and semipositone and may be singular at $u=0$, in which $\mathbf{R}_{0}^{+}=(0,+\infty), \mathbf{R}=$ $(-\infty,+\infty)$. In the present work, we investigate the existence of positive solutions for fractional $q$-derivatives integral boundary value problem (7) and (8) involving the RiemannLiouville's fractional derivative, which is different from [11]. We gave the corresponding Green's function of the boundary value problem (7) and (8), gave some properties of Green's function, and constructed a cone by properties of Green's function. Moreover the existence of at least two and three positive solutions to the boundary value problem (7) and (8) is enunciated.

\section{Preliminaries on $q$-Calculus and Lemmas}

For the convenience of the reader, below we recall some known facts on fractional $q$-calculus. The presentation here can be found in, for example, $[1,3,12,19,21,22]$.

Let $q \in(0,1)$ and define

$$
[a]_{q}=\frac{1-q^{a}}{1-q}, \quad a \in \mathbf{R} .
$$

The $q$-analogue of the power function $(a-b)^{n}$ with $n \in \mathbf{N}_{0}:=$ $\{0,1,2, \ldots\}$ is

$$
\begin{array}{r}
(a-b)^{(0)}=1, \quad(a-b)^{(n)}=\prod_{k=0}^{n-1}\left(a-b q^{k}\right), \\
n \in \mathbf{N}, a, b \in \mathbf{R} .
\end{array}
$$

More generally, if $\gamma \in \mathbf{R}$, then

$$
(a-b)^{(\gamma)}=a^{\gamma} \prod_{k=0}^{\infty} \frac{a-b q^{k}}{a-b q^{\gamma+k}}, \quad a \neq 0 .
$$

Clearly, if $b=0$ then $a^{(\gamma)}=a^{\gamma}$. The $q$-gamma function is defined by

$$
\Gamma_{q}(x)=\frac{(1-q)^{(x-1)}}{(1-q)^{x-1}}, \quad x \in \mathbf{R} \backslash\{0,-1,-2, \ldots\},
$$

and satisfies $\Gamma_{q}(x+1)=[x]_{q} \Gamma_{q}(x)$.

The $q$-derivative of a function $f$ is defined by

$$
\begin{aligned}
& \left(D_{q} f\right)(x)=\frac{f(q x)-f(x)}{(q-1) x}, \\
& \left(D_{q} f\right)(0)=\lim _{x \rightarrow 0}\left(D_{q} f\right)(x),
\end{aligned}
$$

and the $q$-derivatives of higher order by

$$
\begin{gathered}
\left(D_{q}^{0} f\right)(x)=f(x), \\
\left(D_{q}^{n} f\right)(x)=D_{q}\left(D_{q}^{n-1} f\right)(x), \quad n \in \mathbf{N} .
\end{gathered}
$$


The $q$-integral of a function $f$ defined in the interval $[0, b]$ is given by

$$
\begin{array}{r}
\left(I_{q} f\right)(x)=\int_{0}^{x} f(s) d_{q} s=x(1-q) \sum_{k=0}^{\infty} f\left(x q^{k}\right) q^{k}, \\
x \in[0, b] .
\end{array}
$$

If $a \in[0, b]$ and $f$ is defined in the interval $[0, b]$, then its integral from $a$ to $b$ is defined by

$$
\int_{a}^{b} f(s) d_{q} s=\int_{0}^{b} f(s) d_{q} s-\int_{0}^{a} f(s) d_{q} s .
$$

Similar to that for derivatives, an operator $I_{q}^{n}$ is given by

$$
\begin{gathered}
\left(I_{q}^{0} f\right)(x)=f(x), \\
\left(I_{q}^{n} f\right)(x)=I_{q}\left(I_{q}^{n-1} f\right)(x), \quad n \in \mathbf{N} .
\end{gathered}
$$

The fundamental theorem of calculus applies to these operators $I_{q}$ and $D_{q}$, that is,

$$
\left(D_{q} I_{q} f\right)(x)=f(x)
$$

and if $f$ is continuous at $x=0$, then

$$
\left(I_{q} D_{q} f\right)(x)=f(x)-f(0) .
$$

The following formulas will be used later, namely, the integration by parts formula:

$$
\begin{gathered}
\int_{0}^{x} f(s)\left(D_{q} g\right)(s) d_{q} s \\
=[f(s) g(s)]_{s=0}^{s=x}-\int_{0}^{x}\left(D_{q} f\right)(s) g(q s) d_{q} s, \\
{[a(t-s)]^{(\gamma)}=a^{\gamma}(t-s)^{(\gamma)},} \\
{ }_{t} D_{q}(t-s)^{(\gamma)}=[\gamma]_{q}(t-s)^{(\gamma-1)}, \\
{ }_{s} D_{q}(t-s)^{(\gamma)}=[\gamma]_{q}(t-q s)^{(\gamma-1)}, \\
\left({ }_{x} D_{q} \int_{0}^{x} f(x, s) d_{q} s\right)(x)=\int_{0}^{x}{ }_{x} D_{q} f(x, s) d_{q} s+f(q x, x),
\end{gathered}
$$

where ${ }_{t} D_{q}$ denotes the derivative with respect to the variable $t$.

Definition 1. Let $\alpha \geq 0$ and $f$ be a function defined on $[0,1]$. The fractional $q$-integral of Riemann-Liouville type is $\left(I_{q}^{0} f\right)(x)=f(x)$ and

$$
\begin{array}{r}
\left(I_{q}^{\alpha} f\right)(x)=\frac{1}{\Gamma_{q}(\alpha)} \int_{0}^{x}(x-q s)^{(\alpha-1)} f(s) d_{q} s \\
\alpha>0, x \in[0,1] .
\end{array}
$$

The fractional $q$-derivative of order $\alpha \geq 0$ is defined by $\left(D_{q}^{0} f\right)(x)=f(x)$ and $\left(D_{q}^{\alpha} f\right)(x)=\left(D_{q}^{k} I_{q}^{k-\alpha} f\right)(x)$ for $\alpha>0$, where $k$ is the smallest integer greater than or equal to $\alpha$.
Remark 2. Let $\alpha \geq 0$ and $u, v$ be two functions defined on $[0,1]$, then $D_{q}^{\alpha}(u(t)+v(t))=D_{q}^{\alpha} u(t)+D_{q}^{\alpha} v(t)$.

Lemma 3. Assume that $\gamma \geq 0$ and $a \leq b \leq t$, then $(t-a)^{(\gamma)} \geq$ $(t-b)^{(\gamma)}$

Lemma 4. Let $\alpha, \beta \geq 0$ and $f$ be a function defined in $[0,1]$. Then, the following formulas hold:

$$
\begin{aligned}
& \text { (1) }\left(I_{q}^{\beta} I_{q}^{\alpha} f\right)(x)=\left(I_{q}^{\alpha+\beta} f\right)(x), \\
& \text { (2) }\left(D_{q}^{\alpha} I_{q}^{\alpha} f\right)(x)=f(x) .
\end{aligned}
$$

Lemma 5 (see [17]). Let $\alpha>0$ and $n$ be a positive integer. Then, the following equality holds:

$$
\begin{aligned}
\left(I_{q}^{\alpha} D_{q}^{n} f\right)(x)= & \left(D_{q}^{n} I_{q}^{\alpha} f\right)(x) \\
& -\sum_{k=0}^{n-1} \frac{x^{\alpha-n+k}}{\Gamma_{q}(\alpha+k-n+1)}\left(D_{q}^{k} f\right)(0) .
\end{aligned}
$$

Lemma 6. Let $y \in C[0,1], q \in(0,1), 2<\alpha \leq 3,1<\mu<$ $[\alpha]_{q}$. Then the unique solution of the equation

$$
\left(D_{q}^{\alpha} u\right)(t)+y(t)=0, \quad t \in(0,1)
$$

subject to $B C(8)$ is given by

$$
u(t)=\int_{0}^{1} G(t, q s) y(s) d_{q} s
$$

where

$$
G(t, s)=\left\{\begin{array}{l}
\left(t^{\alpha-1}(1-s)^{(\alpha-1)}\left([\alpha]_{q}-\mu+\mu q^{\alpha-1} s\right)\right. \\
\left.-\left([\alpha]_{q}-\mu\right)(t-s)^{(\alpha-1)}\right) \\
\times\left(\left([\alpha]_{q}-\mu\right) \Gamma_{q}(\alpha)\right)^{-1}, \quad 0 \leq s \leq t \leq 1, \\
\left(t^{\alpha-1}(1-s)^{(\alpha-1)}\left([\alpha]_{q}-\mu+\mu q^{\alpha-1} s\right)\right) \\
\quad \times\left(\left([\alpha]_{q}-\mu\right) \Gamma_{q}(\alpha)\right)^{-1}, \quad 0 \leq t \leq s \leq 1 .
\end{array}\right.
$$

Proof. Let us put $n=3$. In view of Definition 1 and Lemma 4, we see that

$$
\left(D_{q}^{\alpha} u\right)(t)=-y(t) \Longleftrightarrow\left(I_{q}^{\alpha} D_{q}^{3} I_{q}^{3-\alpha} u\right)(t)=-\left(I_{q}^{\alpha} y\right)(t) .
$$

Then, it follows from Lemma 5 that the solution $u(t)$ of (25) and $\mathrm{BC}(8)$ is given by

$$
u(t)=c_{1} t^{\alpha-1}+c_{2} t^{\alpha-2}+c_{3} t^{\alpha-3}-\int_{0}^{t} \frac{(t-q s)^{(\alpha-1)}}{\Gamma_{q}(\alpha)} y(s) d_{q} s
$$

for some constants $c_{1}, c_{2}, c_{3} \in \mathbf{R}$. Since $u(0)=0$, we have $c_{3}=$ 0 . Differentiating both sides of (29) and with the help of (20) and (22), we obtain

$$
\begin{aligned}
\left(D_{q} u\right)(t)= & {[\alpha-1]_{q} c_{1} t^{\alpha-2}+[\alpha-2]_{q} c_{2} t^{\alpha-3} } \\
& -\int_{0}^{t} \frac{[\alpha-1]_{q}(t-q s)^{(\alpha-2)}}{\Gamma_{q}(\alpha)} y(s) d_{q} s .
\end{aligned}
$$


Then by the boundary conditions $\left(D_{q} u\right)(0)=0$, we get $c_{2}=0$. Thus, (29) reduces to

$$
u(t)=c_{1} t^{\alpha-1}-\int_{0}^{t} \frac{(t-q s)^{(\alpha-1)}}{\Gamma_{q}(\alpha)} y(s) d_{q} s .
$$

Using the boundary condition $u(1)=\mu \int_{0}^{1} u(s) d_{q} s$, we get

$$
c_{1}=\mu \int_{0}^{1} u(s) d_{q} s+\int_{0}^{1} \frac{(1-q s)^{(\alpha-1)}}{\Gamma_{q}(\alpha)} y(s) d_{q} s .
$$

Hence, we have

$$
\begin{aligned}
u(t)= & t^{\alpha-1}\left(\mu \int_{0}^{1} u(s) d_{q} s+\int_{0}^{1} \frac{(1-q s)^{(\alpha-1)}}{\Gamma_{q}(\alpha)} y(s) d_{q} s\right) \\
& -\int_{0}^{t} \frac{(t-q s)^{(\alpha-1)}}{\Gamma_{q}(\alpha)} y(s) d_{q} s .
\end{aligned}
$$

Integrate the above equation (33) from 0 to 1 , and using (11), (19) and (20), we obtain

$$
\begin{aligned}
\int_{0}^{1} u(t) d_{q} t= & \int_{0}^{1} t^{\alpha-1}\left(\mu \int_{0}^{1} u(s) d_{q} s\right) d_{q} t \\
& +\int_{0}^{1} t^{\alpha-1}\left(\int_{0}^{1} \frac{(1-q s)^{(\alpha-1)}}{\Gamma_{q}(\alpha)} y(s) d_{q} s\right) d_{q} t \\
& -\int_{0}^{1}\left(\int_{0}^{t} \frac{(t-q s)^{(\alpha-1)}}{\Gamma_{q}(\alpha)} y(s) d_{q} s\right) d_{q} t \\
= & \frac{1-q) \mu}{1-q^{\alpha}} \int_{0}^{1} u(s) d_{q} s \\
& +\frac{1-q}{1-q^{\alpha} \int_{0}^{1} \frac{(1-q s)^{(\alpha-1)}}{\Gamma_{q}(\alpha)} y(s) d_{q} s} \\
& -\int_{0}^{1} \frac{(1-q s)^{(\alpha)}}{[\alpha]_{q} \Gamma_{q}(\alpha)} y(s) d_{q} s \\
= & \frac{\mu}{[\alpha]_{q}} \int_{0}^{1} u(s) d_{q} s \\
& +\int_{0}^{1} \frac{(1-q s)^{(\alpha-1)} q^{\alpha} s}{[\alpha]_{q} \Gamma_{q}(\alpha)} y(s) d_{q} s \\
&
\end{aligned}
$$

then

$$
\int_{0}^{1} u(t) d_{q} t=\frac{q^{\alpha}}{[\alpha]_{q}-\mu} \int_{0}^{1} \frac{(1-q s)^{(\alpha-1)} s}{\Gamma_{q}(\alpha)} y(s) d_{q} s
$$

Combining this with (29) and (31) yields

$$
\begin{aligned}
u(t)= & \frac{\mu q^{\alpha}}{\left([\alpha]_{q}-\mu\right) \Gamma_{q}(\alpha)} \int_{0}^{1} t^{\alpha-1}(1-q s)^{(\alpha-1)} s y(s) d_{q} s \\
& +\frac{1}{\Gamma_{q}(\alpha)} \int_{0}^{1} t^{\alpha-1}(1-q s)^{(\alpha-1)} y(s) d_{q} s \\
& -\frac{1}{\Gamma_{q}(\alpha)} \int_{0}^{t}(t-q s)^{(\alpha-1)} y(s) d_{q} s \\
= & \int_{0}^{1} \frac{[\alpha]_{q}-\mu+\mu q^{\alpha} s}{\left([\alpha]_{q}-\mu\right) \Gamma_{q}(\alpha)} \cdot t^{\alpha-1}(1-q s)^{(\alpha-1)} y(s) d_{q} s \\
& -\frac{1}{\Gamma_{q}(\alpha)} \int_{0}^{t}(t-q s)^{(\alpha-1)} y(s) d_{q} s \\
= & \int_{0}^{1} G(t, q s) y(s) d_{q} s .
\end{aligned}
$$

This completes the proof of the lemma.

Remark 7. For the special case where $\mu=0$, Lemmas 6 has been obtained by Ferreira [18].

Lemma 8. The function $G(t, s)$ defined by (27) satisfies the following conditions:

(i) $G(t, s)$ is a continuous function on $(t, s) \in[0,1] \times$ $[0,1]$, and $G(t, q s) \geq 0$, for $(t, s) \in[0,1] \times[0,1]$;

(ii) $\mu q^{\alpha} t^{\alpha-1} \rho(s) \leq\left([\alpha]_{q}-\mu\right) \Gamma_{q}(\alpha) G(t, q s) \leq \lambda t^{\alpha-1},\left([\alpha]_{q}-\right.$ $\mu) \Gamma_{q}(\alpha) G(t, q s) \leq \lambda \rho(s)$, for $(t, s) \in[0,1] \times[0,1]$, where

$$
\begin{gathered}
\rho(s)=(1-q s)^{(\alpha-1)} s, \\
\lambda=\max \left\{[\alpha-1]_{q}\left([\alpha]_{q}-\mu\right)+\mu q^{\alpha}, q^{\alpha-1}[\alpha]_{q}\right\} .
\end{gathered}
$$

Proof. The continuity of $G$ is easily checked. On the other hand, when $0 \leq q s \leq t \leq 1$, in view of Lemma 3 , we have

$$
\begin{aligned}
\left([\alpha]_{q}-\mu\right) \Gamma_{q}(\alpha) G(t, q s) & \\
= & t^{\alpha-1}(1-q s)^{(\alpha-1)}\left([\alpha]_{q}-\mu+\mu q^{\alpha} s\right) \\
& -\left([\alpha]_{q}-\mu\right)(t-q s)^{(\alpha-1)} \\
= & t^{\alpha-1}(1-q s)^{(\alpha-1)}\left([\alpha]_{q}-\mu+\mu q^{\alpha} s\right) \\
& -\left([\alpha]_{q}-\mu\right) t^{\alpha-1}\left(1-q \frac{s}{t}\right)^{(\alpha-1)} \\
\geq & t^{\alpha-1}(1-q s)^{(\alpha-1)}\left([\alpha]_{q}-\mu+\mu q^{\alpha} s\right) \\
& -\left([\alpha]_{q}-\mu\right) t^{\alpha-1}(1-q s)^{(\alpha-1)} \\
= & \mu q^{\alpha} t^{\alpha-1}(1-q s)^{(\alpha-1)} s=\mu q^{\alpha} t^{\alpha-1} \rho(s) \geq 0 .
\end{aligned}
$$


Further, since we have

$$
\begin{aligned}
& G(t, q s)=\left(\left([\alpha]_{q}-\mu\right)\left[t^{\alpha-1}(1-q s)^{(\alpha-1)}-(t-q s)^{(\alpha-1)}\right]\right. \\
& \left.+\mu q^{\alpha} t^{\alpha-1}(1-q s)^{(\alpha-1)} s\right) \\
& \times\left(\left([\alpha]_{q}-\mu\right) \Gamma_{q}(\alpha)\right)^{-1} \\
& =\left(\left([\alpha]_{q}-\mu\right)\left[(t-t q s)^{(\alpha-1)}-(t-q s)^{(\alpha-1)}\right]\right. \\
& \left.+\mu q^{\alpha} t^{\alpha-1}(1-q s)^{(\alpha-1)} s\right) \\
& \times\left(\left([\alpha]_{q}-\mu\right) \Gamma_{q}(\alpha)\right)^{-1} \\
& =\left(\left([\alpha]_{q}-\mu\right) \int_{t-q s}^{t-t q s} D_{q} x^{(\alpha-1)} d_{q} x\right. \\
& \left.+\mu q^{\alpha} t^{\alpha-1}(1-q s)^{(\alpha-1)} s\right) \\
& \times\left(\left([\alpha]_{q}-\mu\right) \Gamma_{q}(\alpha)\right)^{-1} \\
& \leq\left([\alpha-1]_{q}\left([\alpha]_{q}-\mu\right)(t-t q s)^{(\alpha-2)}\right. \\
& \left.\times[(t-t q s)-(t-q s)]+\mu q^{\alpha} t^{\alpha-1}(1-q s)^{(\alpha-1)} s\right) \\
& \times\left(\left([\alpha]_{q}-\mu\right) \Gamma_{q}(\alpha)\right)^{-1} \\
& =\left(q[\alpha-1]_{q}\left([\alpha]_{q}-\mu\right) t^{\alpha-2}(1-q s)^{(\alpha-2)} s(1-t)\right. \\
& \left.+\mu q^{\alpha} t^{\alpha-1}(1-q s)^{(\alpha-1)} s\right) \\
& \times\left(\left([\alpha]_{q}-\mu\right) \Gamma_{q}(\alpha)\right)^{-1} \\
& \leq\left(q[\alpha-1]_{q}\left([\alpha]_{q}-\mu\right) t^{\alpha-2}(1-q s)^{(\alpha-2)} s(1-q s)\right. \\
& \left.+\mu q^{\alpha} t^{\alpha-1}(1-q s)^{(\alpha-1)} s\right) \\
& \times\left(\left([\alpha]_{q}-\mu\right) \Gamma_{q}(\alpha)\right)^{-1} \\
& \leq\left(q[\alpha-1]_{q}\left([\alpha]_{q}-\mu\right) t^{\alpha-2}(1-q s)^{(\alpha-2)} s\left(1-q^{\alpha} s\right)\right. \\
& \left.+\mu q^{\alpha} t^{\alpha-1}(1-q s)^{(\alpha-1)} s\right) \\
& \times\left(\left([\alpha]_{q}-\mu\right) \Gamma_{q}(\alpha)\right)^{-1} \\
& =\left(q[\alpha-1]_{q}\left([\alpha]_{q}-\mu\right) t^{\alpha-2}(1-q s)^{(\alpha-1)} s\right. \\
& \left.+\mu q^{\alpha} t^{\alpha-1}(1-q s)^{(\alpha-1)} s\right) \\
& \times\left(\left([\alpha]_{q}-\mu\right) \Gamma_{q}(\alpha)\right)^{-1},
\end{aligned}
$$

we get

$$
\begin{aligned}
&\left([\alpha]_{q}-\mu\right) \Gamma_{q}(\alpha) G(t, q s) \\
& \leq\left(q[\alpha-1]_{q}\left([\alpha]_{q}-\mu\right)+\mu q^{\alpha}\right)(1-q s)^{(\alpha-1)} s \\
& \leq \lambda \rho(s) \\
&\left([\alpha]_{q}-\mu\right) \Gamma_{q}(\alpha) G(t, q s) \\
& \leq {[\alpha-1]_{q}\left([\alpha]_{q}-\mu\right) t^{\alpha-1}(1-q s)^{(\alpha-1)} } \\
&+\mu q^{\alpha} t^{\alpha-1}(1-q s)^{(\alpha-1)} s \\
& \leq\left([\alpha-1]_{q}\left([\alpha]_{q}-\mu\right)(1-q s)^{(\alpha-1)}\right. \\
&\left.+\mu q^{\alpha}(1-q s)^{(\alpha-1)} s\right) t^{\alpha-1} \\
& \leq\left([\alpha-1]_{q}\left([\alpha]_{q}-\mu\right)+\mu q^{\alpha}\right) t^{\alpha-1} \leq \lambda t^{\alpha-1} .
\end{aligned}
$$

When $0 \leq t \leq q s \leq 1$, since $\mu<[\alpha]_{q}$, we have

$$
\begin{aligned}
& \left([\alpha]_{q}-\mu\right) \Gamma_{q}(\alpha) G(t, q s) \\
& \quad=t^{\alpha-1}(1-q s)^{(\alpha-1)}\left([\alpha]_{q}-\mu+\mu q^{\alpha} s\right) \\
& \quad \geq \mu q^{\alpha} t^{\alpha-1}(1-q s)^{(\alpha-1)} s=\mu q^{\alpha} t^{\alpha-1} \rho(s), \\
& \left([\alpha]_{q}-\mu\right) \Gamma_{q}(\alpha) G(t, q s) \\
& \quad=t^{\alpha-1}(1-q s)^{(\alpha-1)}\left([\alpha]_{q}-\mu+\mu q^{\alpha} s\right) \\
& \quad \leq q^{\alpha-1}[\alpha]_{q} s^{\alpha-1}(1-q s)^{(\alpha-1)} \leq \lambda \rho(s) .
\end{aligned}
$$

This completes the proof of the lemma.

Remark 9. If we let $0<\tau<1$, then

$$
\min _{t \in[\tau, 1]} G(t, q s) \geq \frac{\mu q^{\alpha} \tau^{\alpha-1}}{\left([\alpha]_{q}-\mu\right) \Gamma_{q}(\alpha)} \rho(s), \quad \text { for } s \in[0,1] .
$$

According to [16], we may take $\tau=q^{n}, n \in \mathbf{N}$.

Lemma 10. Let $p \in C[0,1]$. Then the boundary value problem

$$
\begin{gathered}
\left(D_{q}^{\alpha} u\right)(t)+p(t)=0, \quad t \in(0,1), 2<\alpha \leq 3, \\
u(0)=\left(D_{q} u\right)(0)=0, \quad u(1)=\mu \int_{0}^{1} u(s) d_{q} s,
\end{gathered}
$$

has a unique solution $w(t)=\int_{0}^{1} G(t, q s) p(s) d_{q} s$ with

$$
\begin{aligned}
w(t) & \leq \frac{\lambda}{\left([\alpha]_{q}-\mu\right) \Gamma_{q}(\alpha)} t^{\alpha-1} \int_{0}^{1} p(s) d_{q} s \\
& =\lambda_{0} t^{\alpha-1} \int_{0}^{1} p(s) d_{q} s
\end{aligned}
$$

where $\lambda_{0}=\lambda /\left([\alpha]_{q}-\mu\right) \Gamma_{q}(\alpha)$. 
From [39, Theorem 2.3.1], one has the following definition. Let $Q$ be a retract of real Banach space $E, \Omega$ be a relatively bounded open subset of $Q, T: \Omega \rightarrow Q$ be completely continuous operator. The integer $i(T, \Omega, Q)$ be defined by

$$
i(T, \Omega, Q)=\operatorname{deg}\left(I-T \cdot \chi, B(\theta, r) \cap \chi^{-1}(\Omega), \theta\right),
$$

where $\chi: E \rightarrow Q$ is an arbitrary retraction and $r>0$ such that $B(\theta, r) \supset \Omega$. Then the integer $i(T, \Omega, Q)$ is called the fixed point index of $T$ on $\Omega$ with respect to $Q$.

Lemma 11 (see [39]). Let $P$ be a cone in a Banach space E. Let $\Omega$ be an open bounded subset of $E$ with $\Omega_{r}=\Omega \cap P \neq \phi$ and $\bar{\Omega}_{r} \neq P$. Assume that $T: \bar{\Omega}_{r} \rightarrow P$ is a compact map such that $u \neq T u$ for $u \in \partial \Omega_{r}$. Then

(i) if $T\left(\bar{\Omega}_{r}\right) \subset \Omega_{r}$ for $u \in \Omega_{r}$, then $i\left(T, \Omega_{r}, P\right)=1$;

(ii) if there exists $u_{0} \in P \backslash\{\theta\}$ such that $u \neq T u+\lambda u_{0}$ for all $u \in \partial \Omega_{r}$ and $\lambda>0$, then $i\left(T, \Omega_{r}, P\right)=0$.

\section{The Main Results}

In order to abbreviate our discussion, we give the following assumptions.

(H0) There exists $p(t) \in \mathbf{C}[0,1]$, such that

$$
\phi(t) h_{0}(u) \leq f(t, u)+p(t) \leq \varphi(t)(g(u)+h(u))
$$

for all $(t, u) \in[0,1] \times \mathbf{R}_{0}^{+}$, where $\phi, \varphi \in \mathbf{C}\left([0,1], \mathbf{R}_{0}^{+}\right), g \in$ $\mathbf{C}\left(\mathbf{R}_{0}^{+}, \mathbf{R}_{0}^{+}\right)$, and $g(u)$ is nonincreasing with respect to $u ; h_{0}$, $h \in\left(\mathbf{R}^{+}, \mathbf{R}^{+}\right)$and $h_{0}(u), h(u)$ are nondecreasing with respect to $u$, where $\mathbf{R}^{+}=[0,+\infty)$.

$$
(H 1) 0<\int_{0}^{1} \varphi(s) \rho(s) d_{q} s<+\infty .
$$

Let $X=C[0,1]$ be the Banach space endowed with morm $\|u\|=\max _{t \in[0,1]}|u(t)|$, and define the cone $\Omega \in X$ by

$$
\Omega=\left\{u \in X: u(t) \geq \frac{\mu q^{\alpha} t^{\alpha-1}}{\lambda}\|u\|_{X}, q \in(0,1), 2<\alpha \leq 3\right\} .
$$

By a positive solution of BVP (7) and (8), we mean a function $u \in C[0,1]$ such that $u(t)$ satisfies $(7)$ and $(8)$ and $u(t)>0$ on $(0,1)$.

Setting $F(t, u)=f(t, u)+p(t)$ and for any $u \in \Omega, m \in N$, we consider the following singular nonlinear boundary value problem:

$$
\begin{aligned}
& \left(D_{q}^{\alpha} u\right)(t)+F\left(t,[u(t)]^{*}+\frac{1}{m}\right)=0, \quad t \in(0,1), \\
& u(0)=\left(D_{q} u\right)(0)=0, \quad u(1)=\mu \int_{0}^{1} u(s) d_{q} s,
\end{aligned}
$$

where $[u(t)]^{*}=\max \{u(t)-w(t), 0\}$.

According to Remark 2, we can see that if $u(t) \geq w(t)$ for $t \in[0,1]$ is a positive solution of $\operatorname{BVP}(48)$, then $u-w$ is a positive solution of BVP (7) and (8).
Lemma 12. For any $m \in N$, let $T_{m}: \Omega \rightarrow \Omega$ be the operator defined by

$$
\left(T_{m} u\right)(t)=\int_{0}^{1} G(t, q s) F\left(s,[u(s)]^{*}+\frac{1}{m}\right) d_{q} s .
$$

Then $T_{m}: \Omega \rightarrow \Omega$ is completely continuous.

Proof. For any $u \in \Omega, m \in N$, Lemma 8 implies that $\left(T_{m} u\right)(t) \geq 0$ on $[0,1]$, and

$$
\begin{aligned}
& \left(T_{m} u\right)(t) \\
& \quad \geq \frac{\mu q^{\alpha} t^{\alpha-1}}{\left([\alpha]_{q}-\mu\right) \Gamma_{q}(\alpha)} \int_{0}^{1} \rho(s) F\left(s,[u(s)]^{*}+\frac{1}{m}\right) d_{q} s .
\end{aligned}
$$

On the other hand,

$$
\begin{aligned}
\left\|T_{m} u\right\|_{X} & =\max _{0 \leq t \leq 1}\left(T_{m} u\right)(t) \\
& \leq \frac{\lambda}{\left([\alpha]_{q}-\mu\right) \Gamma_{q}(\alpha)} \int_{0}^{1} \rho(s) F\left(s,[u(s)]^{*}+\frac{1}{m}\right) d_{q} s .
\end{aligned}
$$

Then $\left(T_{m} u\right)(t) \geq\left(\mu q^{\alpha} t^{\alpha-1} / \lambda\right)\left\|T_{m} u\right\|_{X}$, which leads to $T_{m}(\Omega) \subset \Omega$. Thus $T_{m}: \Omega \rightarrow \Omega$.

It follows from the nonnegativeness and continuity of $G(t, s)$ and $F$ that the operator $T_{m}$ is continuous. Suppose $B \subset \Omega$ is any bounded set; then, for any $u \in B$, there is a constant number $M>0$ such that $\|u\|_{X} \leq M$. Let

$$
L\left(m^{-1}, M, \lambda_{0}\right)=\lambda_{0}\left[g\left(\frac{1}{m}\right)+h(M+1)\right] .
$$

for all $u \in B$, by Lemma 8 , we have

$$
\begin{aligned}
\left|\left(T_{m} u\right)(t)\right| & \leq \int_{0}^{1}\left|G(t, q s) F\left(s,[u(s)]^{*}+\frac{1}{m}\right)\right| d_{q} s \\
& \leq \lambda_{0} \int_{0}^{1} \rho(s)\left|F\left(s,[u(s)]^{*}+\frac{1}{m}\right)\right| d_{q} s \\
& \leq \lambda_{0} \int_{0}^{1} \rho(s) \varphi(s)\left(g\left([u(s)]^{*}+\frac{1}{m}\right)\right. \\
& \left.+h\left([u(s)]^{*}+\frac{1}{m}\right)\right) d_{q} s \\
& \leq L\left(m^{-1}, M, \lambda_{0}\right) \int_{0}^{1} \rho(s) \varphi(s) d_{q} s<+\infty .
\end{aligned}
$$

Hence, $T_{m}(B)$ is bounded.

On the other hand, for any $\varepsilon>0$, according to $(H 1)$, there is a constant $\delta>0$ such that

$$
\begin{aligned}
& \int_{0}^{\delta} \varphi(s) \rho(s) d_{q} s<\frac{\varepsilon}{6 L\left(m^{-1}, M, \lambda_{0}\right)}, \\
& \int_{1-\delta}^{1} \varphi(s) \rho(s) d_{q} s<\frac{\varepsilon}{6 L\left(m^{-1}, M, \lambda_{0}\right)} .
\end{aligned}
$$


From the property of continuity of $G$, there exists $\eta$ with $0<$ $\eta<\delta$ such that for any $t_{1}, t_{2} \in[0,1]$, and $s \in[0,1]$, when $t_{2}-t_{1}<\eta$ we have

$$
\begin{gathered}
\left|G\left(t_{2}, q s\right)-G\left(t_{1}, q s\right)\right|<\frac{\varepsilon}{3 \varphi_{0} L\left(m^{-1}, M, \lambda_{0}\right)}, \\
\left|\left(T_{m} u\right)\left(t_{2}\right)-\left(T_{m} u\right)\left(t_{1}\right)\right| \\
\leq \int_{0}^{1}\left|G\left(t_{2}, q s\right)-G\left(t_{1}, q s\right)\right| F\left(s,[u(s)]^{*}+\frac{1}{m}\right) d_{q} s \\
\leq L\left(m^{-1}, M, \lambda_{0}\right) \int_{0}^{1}\left|G\left(t_{2}, q s\right)-G\left(t_{1}, q s\right)\right| \varphi(s) d_{q} s \\
=2 L\left(m^{-1}, M, \lambda_{0}\right)\left(\int_{0}^{\delta} \varphi(s) G(s, q s) d_{q} s\right. \\
\left.+\int_{1-\delta}^{1} \varphi(s) G(s, q s) d_{q} s\right) \\
<\frac{2 \varepsilon}{3}+L\left(m^{-1}, M, \lambda_{0}\right) \int_{\delta}^{1-\delta}\left|G\left(t_{2}, q s\right)-G\left(t_{1}, q s\right)\right| \varphi(s) d_{q} s \\
\times \int_{\delta}^{1-\delta}\left|G\left(t_{2}, q s\right)-G\left(t_{1}, q s\right)\right| d_{q} s<\varepsilon,
\end{gathered}
$$

where $\varphi_{0}=\max \{\varphi(t): \delta \leq t \leq 1-\delta\}$. By means of the ArzelaAscoli Theorem, $T_{m}: \Omega \rightarrow \Omega$ is completely continuous.

Theorem 13. Suppose $(\mathrm{H} 0)$ and $(\mathrm{H} 1)$ hold. In addition, assume that the following conditions are satisfied.

(C1) There exists a constant $r>2 \lambda^{2} k_{0} / \mu q^{\alpha}$ such that

$$
\begin{gathered}
\lambda_{0} \int_{0}^{1} \rho(s) \varphi(s)\left(g\left(\frac{\mu q^{\alpha} s^{\alpha-1}}{2 \lambda} r\right)+h(r+1)\right) d_{q} s<r \\
\text { where } k_{0}=\int_{0}^{1} p(s) d_{q} s /\left([\alpha]_{q}-\mu\right) \Gamma_{q}(\alpha) .
\end{gathered}
$$

(C2) There exist constants $\xi_{1}, \xi_{2}$ with $\xi_{2}>\xi_{1}>r$ such that

$$
\mu^{*} h_{0}\left(\frac{1}{2} \xi_{i}\right) \int_{\tau}^{1} \rho(s) \phi(s) d_{q} s>\xi_{i}, \quad i=1,2,
$$

$$
\text { where } \mu^{*}=\mu q^{\alpha} \tau^{\alpha-1} /\left([\alpha]_{q}-\mu\right) \Gamma_{q}(\alpha), \tau=q^{n}, n \in \mathbf{N} .
$$

(C3)

$$
\lim _{u \rightarrow+\infty} \frac{h(u)}{u}=0
$$

Then BVP (7) and (8) has at least two positive solutions $u^{*}, u^{* *}$ with $r \leq\left\|u^{*}\right\|_{X} \leq \xi_{1}<\xi_{2} \leq\left\|u^{* *}\right\|_{X}$.

Proof. First, we prove that

$$
i\left(T_{m}, \Omega_{0}, \Omega\right)=1,
$$

where $\Omega_{0}=\left\{u \in \Omega:\|u\|_{X}<r\right\}$.
To see this, let $u \in \Omega \cap \partial \Omega_{0}$. Then $\|u\|_{X}=r$ and $u(t) \geq$ $\left(\mu q^{\alpha} / \lambda\right) t^{\alpha-1} r$ for $t \in[0,1]$. Now for $t \in(0,1)$, we get

$$
\begin{aligned}
u(t)-w(t) & \geq u(t)-\frac{\lambda}{\left([\alpha]_{q}-\mu\right) \Gamma_{q}(\alpha)} t^{\alpha-1} \int_{0}^{1} p(s) d_{q} s \\
& \geq u(t)-\frac{\mu q^{\alpha}}{2 \lambda} t^{\alpha-1} r \geq \frac{1}{2} u(t) \\
& \geq \frac{\mu q^{\alpha} t^{\alpha-1}}{2 \lambda}\|u\|_{X}=\frac{\mu q^{\alpha} t^{\alpha-1}}{2 \lambda} r .
\end{aligned}
$$

So, for any $u \in \Omega \cap \partial \Omega_{0}, t \in(0,1)$, we get

$$
\frac{\mu q^{\alpha} t^{\alpha-1}}{2 \lambda} r \leq u(t)-w(t) \leq r .
$$

It follows from (C1), (61) and Lemma 8 that, for any $u \in \Omega \cap$ $\partial \Omega_{0}$,

$$
\begin{aligned}
& \left(T_{m} u\right)(t) \\
& =\int_{0}^{1} G(t, q s) F\left(s,[u(s)]^{*}+\frac{1}{m}\right) d_{q} s \\
& \leq \lambda_{0} \int_{0}^{1} \rho(s) \varphi(s)\left(g\left([u(s)]^{*}+\frac{1}{m}\right)\right. \\
& \left.\quad+h\left([u(s)]^{*}+\frac{1}{m}\right)\right) d_{q} s \\
& \leq \lambda_{0} \int_{0}^{1} \rho(s) \varphi(s)\left(g\left(\frac{\mu q^{\alpha} s^{\alpha-1}}{2 \lambda} r\right)+h(r+1)\right) d_{q} s<r \\
& =\|u\|_{X} .
\end{aligned}
$$

This together with (56) yields $T_{m}\left(\bar{\Omega}_{0}\right) \subset \Omega_{0}$. From the (i) of Lemma 11, (59) is satisfied.

Let us choose $\epsilon>0$ such that

$$
\epsilon \lambda_{0} \int_{0}^{1} \rho(s) \varphi(s) d_{q} s<1 .
$$

Then for the above $\epsilon$, according to (C2) and (C3), there exists $R>\xi_{2}>0$ such that, for any $u \geq R$,

$$
h(u) \leq \epsilon u \text {. }
$$

Take

$$
\begin{aligned}
R_{1}=R+ & \left(\lambda_{0} \int_{0}^{1} \rho(s) \varphi(s)\right. \\
& \left.\times\left(g\left(\frac{\mu q^{\alpha} s^{\alpha-1}}{2 \lambda} R\right)+h(R+1)+\epsilon\right) d_{q} s\right) \\
& \times\left(1-\epsilon \lambda_{0} \int_{0}^{1} \rho(s) \varphi(s) d_{q} s\right)^{-1},
\end{aligned}
$$

then $R_{1}>R>\xi_{2}$. 
Now let $\Omega_{1}=\left\{u \in \Omega:\|u\|_{X}<R_{1}\right\}$ and $\partial \Omega_{1}=\{u \in \Omega$ $\left.\|u\|_{X}=R_{1}\right\}$. Then, for any $u \in \Omega \cap \partial \Omega_{1}$, we have

$$
\begin{aligned}
\left(T_{m} u\right)(t)= & \int_{0}^{1} G(t, q s) F\left(s,[u(s)]^{*}+\frac{1}{m}\right) d_{q} s \\
\leq & \lambda_{0} \int_{0}^{1} \rho(s) \varphi(s) \\
& \times\left(g\left([u(s)]^{*}+\frac{1}{m}\right)+h\left([u(s)]^{*}+\frac{1}{m}\right)\right) d_{q} s \\
\leq & \lambda_{0} \int_{0}^{1} \rho(s) \varphi(s) \\
& \times\left(g\left([u(s)]^{*}+\frac{1}{m}\right)\right. \\
& \left.+\max _{u \in[0, R]} h\left([u(s)]^{*}+\frac{1}{m}\right)\right) d_{q} s \\
& +\lambda_{0} \int_{0}^{1} \rho(s) \varphi(s) \epsilon\left(\|u\|_{X}+1\right) d_{q} s \\
\leq & \lambda_{0} \int_{0}^{1} \rho(s) \varphi(s) \\
& \times\left(g\left(\frac{\mu q^{\alpha} s^{\alpha-1}}{2 \lambda} R_{1}\right)+h(R+1)\right) d_{q} s \\
& +\epsilon \lambda_{0} \int_{0}^{1} \rho(s) \varphi(s) d_{q} s \cdot R_{1}<R_{1}=\|u\|_{X} . \\
\leq & \lambda_{0} \int_{0}^{1} \rho(s) \varphi(s) \\
& \times\left(g\left(\frac{\mu q^{\alpha} s^{\alpha-1}}{2 \lambda} R\right)+h(R+1)+\epsilon\right) d_{q} s \\
& (s) \epsilon\left(R_{1}+1\right) d_{q} s \\
& \\
& (s)
\end{aligned}
$$

This together with (56) yields $T_{m}\left(\bar{\Omega}_{1}\right) \subset \Omega_{1}$, which implies that

$$
i\left(T_{m}, \Omega_{1}, \Omega\right)=1 .
$$

In the following, let

$$
\begin{aligned}
& \Omega_{10}=\left\{u \in \Omega:\|u\|<R_{1}, \min _{t \in[\tau, 1]} u(t)>\xi_{1}\right\}, \\
& \Omega_{11}=\left\{u \in \Omega:\|u\|<R_{1}, \min _{t \in[\tau, 1]} u(t)>\xi_{2}\right\} .
\end{aligned}
$$

It is easy to see that $\Omega_{i}, \Omega_{1 i},(i=0,1)$ are bounded sets and satisfy

$$
\Omega_{0} \subset \Omega_{1}, \quad \Omega_{10} \subset \Omega_{1}, \quad \Omega_{11} \subset \Omega_{10}, \quad \Omega_{10} \cap \Omega_{0}=\emptyset .
$$

For any $u \in \Omega \cap \partial \Omega_{10}$, by Remark 9 and (61), we have

$$
u(t)-w(t) \geq \frac{1}{2} u(t) \geq \frac{1}{2} \xi_{1}, \quad \text { for any } t \in[\tau, 1] .
$$

Thus

$$
\begin{aligned}
\min _{t \in[\tau, 1]} & \left(T_{m} u\right)(t) \\
& =\min _{t \in[\tau, 1]} \int_{0}^{1} G(t, q s) F\left(s,[u(s)]^{*}+\frac{1}{m}\right) d_{q} s \\
& \geq \min _{t \in[\tau, 1]} \int_{0}^{1} G(t, q s) \phi(s) h_{0}\left([u(s)]^{*}+\frac{1}{m}\right) d_{q} s \\
& \geq \mu^{*} h_{0}\left(\frac{1}{2} \xi_{1}\right) \int_{\tau}^{1} \rho(s) \phi(s) d_{q} s>\xi_{1} .
\end{aligned}
$$

This means that $T_{m}\left(\bar{\Omega}_{10}\right) \subset \Omega_{10}$, for $u \in \bar{\Omega}_{10}$. Thus, it follows from the (i) of Lemma 11 that

$$
i\left(T_{m}, \Omega_{10}, \Omega\right)=1 .
$$

Similarly, we can prove that, for any $u \in \bar{\Omega}_{11}$,

$$
i\left(T_{m}, \Omega_{11}, \Omega\right)=1 \text {. }
$$

Thus, using (59), (67), and (72), we obtain

$$
\begin{aligned}
i\left(T_{m}, \Omega_{1} \backslash\left(\bar{\Omega}_{0} \cap \bar{\Omega}_{10}\right), \Omega\right) \\
=i\left(T_{m}, \Omega_{1}, \Omega\right)-i\left(T_{m}, \Omega_{0}, \Omega\right) \\
-i\left(T_{m}, \Omega_{10}, \Omega\right)=-1
\end{aligned}
$$

which implies that $T_{m}$ has at least one fixed point $u_{m 1} \in \Omega_{1} \backslash$ $\left(\bar{\Omega}_{0} \cap \bar{\Omega}_{10}\right)$ and satisfies

$$
\begin{array}{r}
T_{m} u_{m 1}(t)=\int_{0}^{1} G(t, q s) F\left(s,\left[u_{m 1}(s)\right]^{*}+\frac{1}{m}\right) d_{q} s, \\
t \in[0,1],
\end{array}
$$

and $r \leq\left\|u_{m 1}\right\|_{X} \leq \xi_{1}$.

Obviously, $F\left(t,\left[u_{m 1}(t)\right]^{*}+1 / m\right)$ is continuous for any $u_{m 1} \in C\left([0,1], \mathbf{R}^{+}\right), t \in(0,1)$. Also, it can be seen that $u_{m 1}$ has uniform lower and upper bounds. This directly comes from $u_{m 1} \in \Omega_{1} \backslash\left(\bar{\Omega}_{0} \cap \bar{\Omega}_{10}\right)$. Hence, in order to pass the solution $u_{m 1}$ of the problem (48) to that of the original problem (7) and (8), we need the following fact:

$$
\left\{u_{m 1}\right\}_{m=1}^{\infty} \text { is an equicontinuous family on }[0,1] \text {. }
$$

As in the proof of Lemma 12, we can prove that the sequence $\left\{u_{m 1}\right\}_{m=1}^{\infty}$ is equicontinuous on $[0,1]$. Now the Arzela-Ascoli Theorem guarantees that the sequence $\left\{u_{m 1}\right\}_{m=1}^{\infty}$ has a subsequence $\left\{u_{m_{k}, 1}\right\}_{k=1}^{\infty}$, converging uniformly on $[0,1]$ to $u_{1} \in X$. As for (61) and the fact $r \leq\left\|u_{m 1}\right\|_{X} \leq \xi_{1}$, we obtain that

$$
\frac{\mu q^{\alpha} t^{\alpha-1}}{2 \lambda} r \leq u_{1}(t)-w(t) \leq \xi_{1}, \quad \forall t \in[0,1] .
$$

Moreover, $u_{m_{k}, 1}$ satisfies the following integral equation:

$$
u_{m_{k}, 1}=\int_{0}^{1} G(t, q s) F\left(s,\left[u_{m_{k}, 1}(s)\right]^{*}+\frac{1}{m_{k}}\right) d_{q} s .
$$


Letting $k \rightarrow+\infty$, we have

$$
u_{1}=\int_{0}^{1} G(t, q s) F\left(s,\left[u_{1}(s)\right]^{*}\right) d_{q} s .
$$

Let $u^{*}(t)=u_{1}(t)-w(t)$, then $u^{*}$ is a positive solution of BVP (7) and (8).

From (73), $T_{m}$ has at least one fixed point $u_{m 2} \in \bar{\Omega}_{11}$. Similar to (76), there exists a subsequence $\left\{u_{m_{k}, 2}\right\}_{k=1}^{\infty} \subseteq$ $\left\{u_{m 2}\right\}_{m=1}^{\infty}$ such that $\lim _{k \rightarrow+\infty} u_{m_{k}, 2}(t)=u_{2}(t) \in X$, and $\xi_{2} \leq$ $\left\|u_{2}\right\|_{X} \leq R_{1}$. Let $u^{* *}(t)=u_{2}(t)-w(t)$, then $u^{* *}$ is also a positive solution of BVP (7) and (8).

Since $\xi_{1}<\xi_{2}$, we have $u^{*} \neq u^{* *}$. This implies that $u^{*}, u^{* *}$ are two different positive solutions of BVP (7) and (8).

Theorem 14. Suppose (H0), (H1), (C1), and (C2) hold. In addition, assume that the following conditions are satisfied.

(C4) There exists $R_{1}$ with $R_{1}>\xi_{2}\left(\xi_{2}\right.$ as given in (C2)) such that

$$
\lambda_{0} \int_{0}^{1} \rho(s) \varphi(s)\left(g\left(\frac{\mu q^{\alpha} s^{\alpha-1}}{2 \lambda} R_{1}\right)+h\left(R_{1}+1\right)\right) d_{q} s<R_{1} \text {. }
$$

(C5)

$$
\lim _{u \rightarrow+\infty} \frac{h_{0}(u)}{u}=+\infty
$$

Then BVP (7) and (8) has at least three different positive solutions.

Proof. It can be seen that condition $(C 4)$ is equivalent to condition (C3). As a consequence we obtain that the BVP (7) and (8) has at least two different positive solutions $u_{1}, u_{2}$ with $r \leq\left\|u_{1}\right\|_{X}<\left\|u_{2}\right\|_{X}<R_{1}$. that

On the other hand, choose a real number $M^{*}>0$ such

$$
M^{*} \geq \frac{2 \lambda}{\mu^{*} \mu q^{\alpha} \tau^{\alpha-1} \int_{\tau}^{1} \rho(s) \phi(s) d_{q} s} .
$$

By (C5), there exists $R_{1}^{*}>R_{1}$ such that

$$
h_{0}(u) \geq M^{*} u, \quad u \geq R_{1}^{*} .
$$

Choose

$$
R_{2}>\max \left\{R_{1}, \frac{2 \lambda R_{1}^{*}}{\mu q^{\alpha} \tau^{\alpha-1}}\right\},
$$

and let $\Omega_{2}=\left\{u \in \Omega:\|u\|_{X}<R_{2}\right\}$.

In the following, we will prove that

$$
u \neq T_{m} u+v \hbar, \quad \hbar \in \Omega \backslash\{\theta\}, \forall u \in \partial \Omega_{2}, v \in[0,1], m \in \mathbf{N} .
$$

Suppose that (85) is false; then there exists $v_{0} \in[0,1], u_{0} \in$ $\partial \Omega_{2}$, such that

$$
u_{0}=T_{m} u_{0}+v_{0} \hbar
$$

For $u_{0} \in \partial \Omega_{2}$ and for any $t \in[\tau, 1]$, we have

$$
\begin{aligned}
u_{0}(t)-w(t) & \geq u_{0}(t)-\frac{\lambda}{\left([\alpha]_{q}-\mu\right) \Gamma_{q}(\alpha)} t^{\alpha-1} \int_{0}^{1} p(s) d_{q} s \\
& \geq\left(1-\frac{\lambda^{2} \int_{0}^{1} \rho(s) d_{q} s}{\mu q^{\alpha}\left([\alpha]_{q}-\mu\right) \Gamma_{q}(\alpha) R_{2}}\right) u_{0}(t) \\
& \geq \frac{1}{2} u_{0}(t) \geq \frac{\mu q^{\alpha} t^{\alpha-1}}{2 \lambda}\left\|u_{0}\right\|_{X} \\
& \geq \frac{\mu q^{\alpha} \tau^{\alpha-1}}{2 \lambda} R_{2}>R_{1}^{*}>0 .
\end{aligned}
$$

It follows from (83) and (85) that we have

$$
\begin{aligned}
R_{2} & =\left\|u_{0}\right\|_{X}=\left\|T_{m} u_{0}+v_{0} \hbar\right\|_{X} \\
& \geq \int_{0}^{1} G(t, q s) F\left(s,\left[u_{0}(s)\right]^{*}+\frac{1}{m}\right) d_{q} s \\
& >\mu^{*} \int_{\tau}^{1} \rho(s) \phi(s) h_{0}\left(\left[u_{0}(s)\right]^{*}+\frac{1}{m}\right) d_{q} s \\
& \geq \mu^{*} \int_{\tau}^{1} \rho(s) \phi(s) d_{q} s \cdot M^{*}\left(\left[u_{0}(s)\right]^{*}+\frac{1}{m}\right) \\
& \geq \frac{M^{*} \mu^{*} \mu q^{\alpha} \tau^{\alpha-1} \int_{\tau}^{1} \rho(s) \phi(s) d_{q} s}{2 \lambda} \cdot R_{2} \geq R_{2},
\end{aligned}
$$

which is a contradiction. Hence, (85) is ture; from the (ii) of Lemma 11, we get

$$
i\left(T_{m}, \Omega_{2}, \Omega\right)=0 \text {. }
$$

Combining this with (67) yields

$$
i\left(T_{m}, \Omega_{2} \backslash \bar{\Omega}_{1}, \Omega\right)=i\left(T_{m}, \Omega_{2}, \Omega\right)-i\left(T_{m}, \Omega_{1}, \Omega\right)=-1 .
$$

This implies that $T_{m}$ has at least one fixed point $u_{m 3} \in$ $\Omega_{2} \backslash \bar{\Omega}_{1}$ with $R_{1}<\left\|u_{m 3}\right\|_{X} \leq R_{2}$. Similar to (76), there exists a subsequence $\left\{u_{m_{k}, 3}\right\}_{k=1}^{\infty} \subseteq\left\{u_{m 3}\right\}_{m=1}^{\infty}$ such that $\lim _{k \rightarrow+\infty} u_{m_{k}, 3}(t)=u_{3}^{*}(t) \in X$, and $R_{1}<\left\|u_{3}^{*}\right\|_{X} \leq R_{2}$. Let $u_{3}(t)=u_{3}^{*}(t)-w(t)$, then $u_{3}$ is also a positive solution of BVP (7) and (8). So the proof is complete.

By the induction method, we can obtain the following multiplicity results for BVP (7) and (8).

Corollary 15. Suppose (H0), (H1), (C1), and (C5) hold. In addition, there exist constants $r_{i}, \xi_{i k},(i=1, \ldots, m ; k=1,2)$ 
with $r<\xi_{11}<\xi_{12}<r_{1}<\xi_{21}<\xi_{22}<r_{2}<\cdots<\xi_{m 1}<\xi_{m 2}<$ $r_{m}$ such that

$$
\begin{array}{r}
\lambda \int_{0}^{1} \rho(s) \varphi(s)\left(g\left(\frac{\mu q^{\alpha} s^{\alpha-1}}{2 \lambda} r_{i}\right)+h\left(r_{i}+1\right)\right) d_{q} s<r_{i} \\
i=1,2, \ldots, m \\
\mu^{*} h_{0}\left(\frac{1}{2} \xi_{i k}\right) \int_{\tau}^{1} \rho(s) \phi(s) d_{q} s>\xi_{i k}, \quad i=1,2, \ldots, m ; \\
k=1,2,
\end{array}
$$

where $\mu^{*}=\mu q^{\alpha} \tau^{\alpha-1} /\left([\alpha]_{q}-\mu\right) \Gamma_{q}(\alpha)$.

Then BVP (7) and (8) has at least $2 m+1$ different positive solutions.

Now we present an example to illustrate our main results.

Example 16. Consider the following problem:

$$
\begin{gathered}
D_{0.5}^{2.5} u(t)+\frac{t}{10}\left(\frac{1}{20 u}+h(u)\right)-40(1+t)=0, \quad 0<t<1, \\
u(0)=\left(D_{0.5} u\right)(0)=0, \quad u(1)=1.5 \int_{0}^{1} u(s) d_{0.5} s
\end{gathered}
$$

where

$$
h(u)= \begin{cases}8 u, & 0 \leq u \leq 60 \\ \frac{1}{490} u^{3}, & 60 \leq u \leq 900 \\ 30 u^{2}, & 900 \leq u \leq 1600 \\ 3.2 \times 10^{5} \sqrt{u}, & u \geq 1600\end{cases}
$$

Then BVP (91) has at least two positive solutions.

Proof. In this case, $\alpha=2.5, q=0.5, \mu=1.5$, and

$$
f(t, u)=\frac{t}{10}\left(\frac{1}{20 u}+h(u)\right)-40(1+t) \text {. }
$$

Let

$$
\begin{gathered}
p(t)=40(1+t), \quad \phi(t)=\frac{t}{10}, \quad \varphi(t)=\frac{\sqrt{t}}{10}, \\
g(u)=\frac{1}{20 u}, \quad h_{0}(u)=\frac{7}{8} h(u) .
\end{gathered}
$$

It is easy to see that the assumptions (H0), $(H 1)$ hold.

Take $n=2$, then $\tau=q^{n}=0.25$. By calculation, we get

$$
\begin{gathered}
\lambda \approx 0.7335, \quad \lambda_{0} \approx 1.3913, \quad \mu^{*} \approx 0.0524 \\
k_{0}=\frac{\int_{0}^{1} p(s) d_{q} s}{\left([\alpha]_{q}-\mu\right) \Gamma_{q}(\alpha)} \approx \frac{\int_{0}^{1} 40(1+s) d_{0.5} s}{0.5272} \approx 12.6454 .
\end{gathered}
$$

Set $r=52$, then

$$
\begin{gathered}
r=52>\frac{2 \lambda^{2} k_{0}}{\mu q^{\alpha}} \approx 51.3086 \\
\Delta:=\lambda_{0} \int_{0}^{1} \rho(s) \varphi(s)\left(g\left(\frac{\mu q^{\alpha} s^{\alpha-1}}{2 \lambda} r\right)+h(r+1)\right) d_{q} s \\
=\frac{\lambda_{0}}{10} \int_{0}^{1}\left(1-\frac{1}{2} s\right)^{(3 / 2)} s^{3 / 2} \\
\leq \frac{\lambda_{0}}{10}\left(\frac{\lambda}{520 \mu q^{\alpha}}+h\left(\frac{\lambda}{10 \mu q^{\alpha} s^{3 / 2} r}+h(r+1)\right) d_{q} s\right. \\
\quad \times \int_{0}^{1}\left(1-\frac{1}{2} s\right)^{(3 / 2)} d_{q} \approx 35.8280<r=52,
\end{gathered}
$$

which implies that the assumption $(C 1)$ holds.

On the other hand, take $\xi_{1}=1 \times 10^{3}, \xi_{2}=1.1 \times 10^{3}$, then

$$
\begin{aligned}
& \mu^{*} h_{0}\left(\frac{1}{2} \xi_{1}\right) \int_{\tau}^{1} \rho(s) \phi(s) d_{q} s \\
& \quad \frac{\mu^{*}}{10} h_{0}\left(\frac{1}{2} \xi_{1}\right) \int_{1 / 4}^{1}\left(1-\frac{1}{2} s\right)^{(3 / 2)} s^{3 / 2} d_{q} s \\
& \geq \frac{\mu^{*}}{160} h_{0}\left(\frac{1}{2} \xi_{1}\right) \int_{1 / 4}^{1}\left(1-\frac{1}{2} s\right)^{(3 / 2)} d_{q} s \\
& \approx 1.1681 \times 10^{3}>1 \times 10^{3}=\xi_{1}, \\
& \mu^{*} h_{0}\left(\frac{1}{2} \xi_{2}\right) \int_{\tau}^{1} \rho(s) \phi(s) d_{q} s \\
& \quad \geq 1.4135 \times 10^{3}>1.1 \times 10^{3}=\xi_{2}
\end{aligned}
$$

which implies that the assumption $(C 2)$ holds.

Finally, we have

$$
\begin{aligned}
\lim _{u \rightarrow+\infty} \frac{h(u)}{u} & =\lim _{u \rightarrow+\infty} \frac{3.2 \times 10^{5} \sqrt{u}}{u} \\
& =\lim _{u \rightarrow+\infty} \frac{3.2 \times 10^{5}}{\sqrt{u}}=0 .
\end{aligned}
$$

Thus (C3) also holds. It follows from Theorem 13 that the BVP (91) has at least two positive solutions $u^{*}, u^{* *}$ with $52 \leq$ $\left\|u^{*}\right\| \leq 1 \times 10^{3}<1.1 \times 10^{3} \leq\left\|u^{* *}\right\|$.

\section{Acknowledgments}

The authors would like to express their thanks to the editor of the journal and the anonymous referees for their carefully reading of the paper. The authors are supported by the National Natural Science Foundation of China (11271372, and 11201138) is also supported by Hunan Provincial Natural Science Foundation of China (12JJ2004), and the Scientific Research Fund of Hunan Provincial Education Department (12B034). 


\section{References}

[1] F. H. Jackson, “q-difference equations," American Journal of Mathematics, vol. 32, no. 4, pp. 305-314, 1910.

[2] R. D. Carmichael, "The general theory of linear $q$-difference equations," American Journal of Mathematics, vol. 34, no. 2, pp. 147-168, 1912.

[3] V. Kac and P. Cheung, Quantum Calculus, Springer, New York, NY, USA, 2002.

[4] R. Finkelstein and E. Marcus, "Transformation theory of the $q$ oscillator," Journal of Mathematical Physics, vol. 36, no. 6, pp. 2652-2672, 1995.

[5] P. G. O. Freund and A. V. Zabrodin, "The spectral problem for the $q$-Knizhnik-Zamolodchikov equation and continuous $q$ Jacobi polynomials," Communications in Mathematical Physics, vol. 173, no. 1, pp. 17-42, 1995.

[6] G.-N. Han and J. Zeng, "On a q-sequence that generalizes the median Genocchi numbers," Annales des Sciences Mathématiques du Québec, vol. 23, no. 1, pp. 63-72, 1999.

[7] R. Floreanini and L. Vinet, "Quantum symmetries of $q$ difference equations," Journal of Mathematical Physics, vol. 36, no. 6, pp. 3134-3156, 1995.

[8] G. Bangerezako, "Variational q-calculus," Journal of Mathematical Analysis and Applications, vol. 289, no. 2, pp. 650-665, 2004.

[9] A. Dobrogowska and A. Odzijewicz, "Second order q-difference equations solvable by factorization method," Journal of Computational and Applied Mathematics, vol. 193, no. 1, pp. 319-346, 2006.

[10] M. E. H. Ismail and P. Simeonov, " $q$-difference operators for orthogonal polynomials," Journal of Computational and Applied Mathematics, vol. 233, no. 3, pp. 749-761, 2009.

[11] B. Ahmad, S. Ntouyas, and I. Purnaras, "Existence results for nonlocal boundary value problems of nonlinear fractional $q$ difference equations," Advances in Difference Equations, vol. 2012, article 140, 2012.

[12] M. El-Shahed and H. A. Hassan, "Positive solutions of $q$ difference equation," Proceedings of the American Mathematical Society, vol. 138, no. 5, pp. 1733-1738, 2010.

[13] M. H. Annaby and Z. S. Mansour, " $q$-Taylor and interpolation series for Jackson $q$-difference operators," Journal of Mathematical Analysis and Applications, vol. 344, no. 1, pp. 472-483, 2008.

[14] B. Ahmad and S. K. Ntouyas, "Boundary value problems for $q$ difference inclusions," Abstract and Applied Analysis, vol. 2011, Article ID 292860, 15 pages, 2011.

[15] G. Gasper and M. Rahman, "Some systems of multivariable orthogonal $q$-Racah polynomials," The Ramanujan Journal, vol. 13, no. 1-3, pp. 389-405, 2007.

[16] H. Gauchman, "Integral inequalities in q-calculus," Computers \& Mathematics with Applications, vol. 47, no. 2-3, pp. 281-300, 2004.

[17] R. A. C. Ferreira, "Nontrivial solutions for fractional $q$ difference boundary value problems," Electronic Journal of Qualitative Theory of Differential Equations, vol. 70, pp. 1-10, 2010.

[18] R. A. C. Ferreira, "Positive solutions for a class of boundary value problems with fractional q-differences," Computers \& Mathematics with Applications, vol. 61, no. 2, pp. 367-373, 2011.

[19] M. El-Shahed and F. M. Al-Askar, "Positive solutions for boundary value problem of nonlinear fractional $q$-difference equation," ISRN Mathematical Analysis, vol. 2011, Article ID 385459, 12 pages, 2011.
[20] S. Liang and J. Zhang, "Existence and uniqueness of positive solutions for three-point boundary value problem with fractional q-differences," Journal of Applied Mathematics and Computing, vol. 40, no. 1-2, pp. 277-288, 2012.

[21] J. R. Graef and L. Kong, "Positive solutions for a class of higher order boundary value problems with fractional q-derivatives," Applied Mathematics and Computation, vol. 218, no. 19, pp. 9682-9689, 2012.

[22] M. H. Annaby and Z. S. Mansour, q-Fractional Calculus and Equations, Springer, Berlin, Germany, 2012.

[23] I. Podlubny, Fractional Differential Equations, vol. 198 of Mathematics in Science and Engineering, Academic Press, London, UK, 1999.

[24] C. F. Li, X. N. Luo, and Y. Zhou, "Existence of positive solutions of the boundary value problem for nonlinear fractional differential equations," Computers \& Mathematics with Applications, vol. 59, no. 3, pp. 1363-1375, 2010.

[25] G. Wang, B. Ahmad, and L. Zhang, "Some existence results for impulsive nonlinear fractional differential equations with mixed boundary conditions," Computers \& Mathematics with Applications, vol. 62, no. 3, pp. 1389-1397, 2011.

[26] B. Ahmad and S. Sivasundaram, "On four-point nonlocal boundary value problems of nonlinear integro-differential equations of fractional order," Applied Mathematics and Computation, vol. 217, no. 2, pp. 480-487, 2010.

[27] S. Staněk, "The existence of positive solutions of singular fractional boundary value problems," Computers \& Mathematics with Applications, vol. 62, no. 3, pp. 1379-1388, 2011.

[28] W.-X. Zhou and Y.-D. Chu, "Existence of solutions for fractional differential equations with multi-point boundary conditions," Communications in Nonlinear Science and Numerical Simulation, vol. 17, no. 3, pp. 1142-1148, 2012.

[29] Y. Zhao, H. Chen, and L. Huang, "Existence of positive solutions for nonlinear fractional functional differential equation," Computers \& Mathematics with Applications, vol. 64, no. 10, pp. 3456-3467, 2012.

[30] X. Zhang, L. Liu, and Y. Wu, "Existence results for multiple positive solutions of nonlinear higher order perturbed fractional differential equations with derivatives," Applied Mathematics and Computation, vol. 219, no. 4, pp. 1420-1433, 2012.

[31] C. S. Goodrich, "On discrete sequential fractional boundary value problems," Journal of Mathematical Analysis and Applications, vol. 385, no. 1, pp. 111-124, 2012.

[32] S. Zhang, "Positive solutions to singular boundary value problem for nonlinear fractional differential equation," Computers \& Mathematics with Applications, vol. 59, no. 3, pp. 1300-1309, 2010.

[33] Y. Zhao, L. Huang, X. Wang, and X. Zhu, "Existence of solutions for fractional integro-differential equation with multipoint boundary value problem in Banach spaces," Abstract and Applied Analysis, vol. 2012, Article ID 172963, 19 pages, 2012.

[34] X. Zhang, L. Liu, and Y. Wu, "The eigenvalue problem for a singular higher order fractional differential equation involving fractional derivatives," Applied Mathematics and Computation, vol. 218, no. 17, pp. 8526-8536, 2012.

[35] Y. Wang, L. Liu, and Y. Wu, "Positive solutions of a fractional boundary value problem with changing sign nonlinearity," Abstract and Applied Analysis, vol. 2012, Article ID 149849, 12 pages, 2012.

[36] W. A. Al-Salam, "Some fractional $q$-integrals and $q$-derivatives," Proceedings of the Edinburgh Mathematical Society, vol. 15, pp. 135-140, 1966-1967. 
[37] R. P. Agarwal, "Certain fractional q-integrals and q-derivatives," Proceedings of the Cambridge Philosophical Society, vol. 66, pp. 365-370, 1969.

[38] P. M. Rajković, S. D. Marinković, and M. S. Stanković, "Fractional integrals and derivatives in q-calculus," Applicable Analysis and Discrete Mathematics, vol. 1, no. 1, pp. 311-323, 2007.

[39] D. J. Guo and V. Lakshmikantham, Nonlinear Problems in Abstract Cones, vol. 5, Academic Press, San Diego, Calif, USA, 1988. 


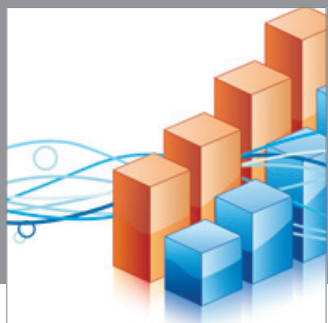

Advances in

Operations Research

mansans

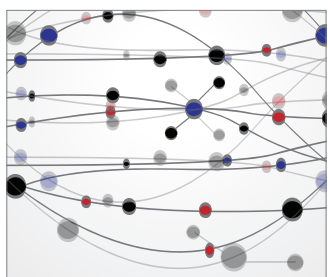

The Scientific World Journal
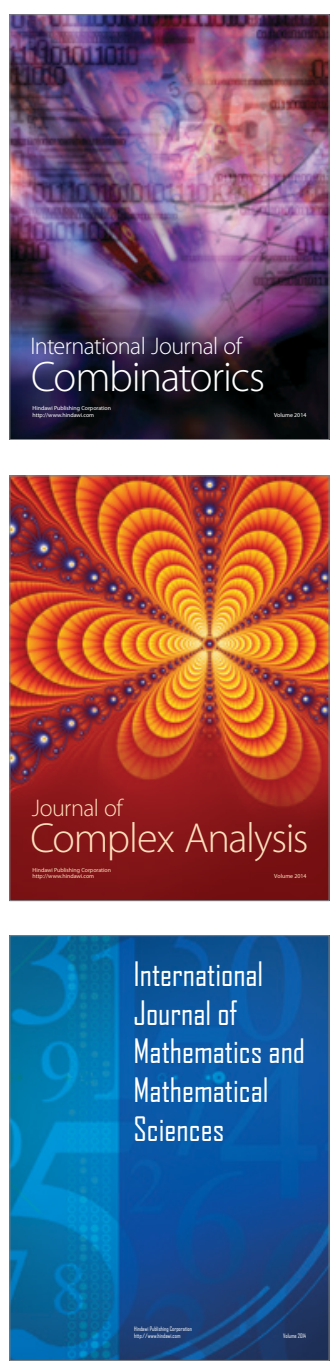
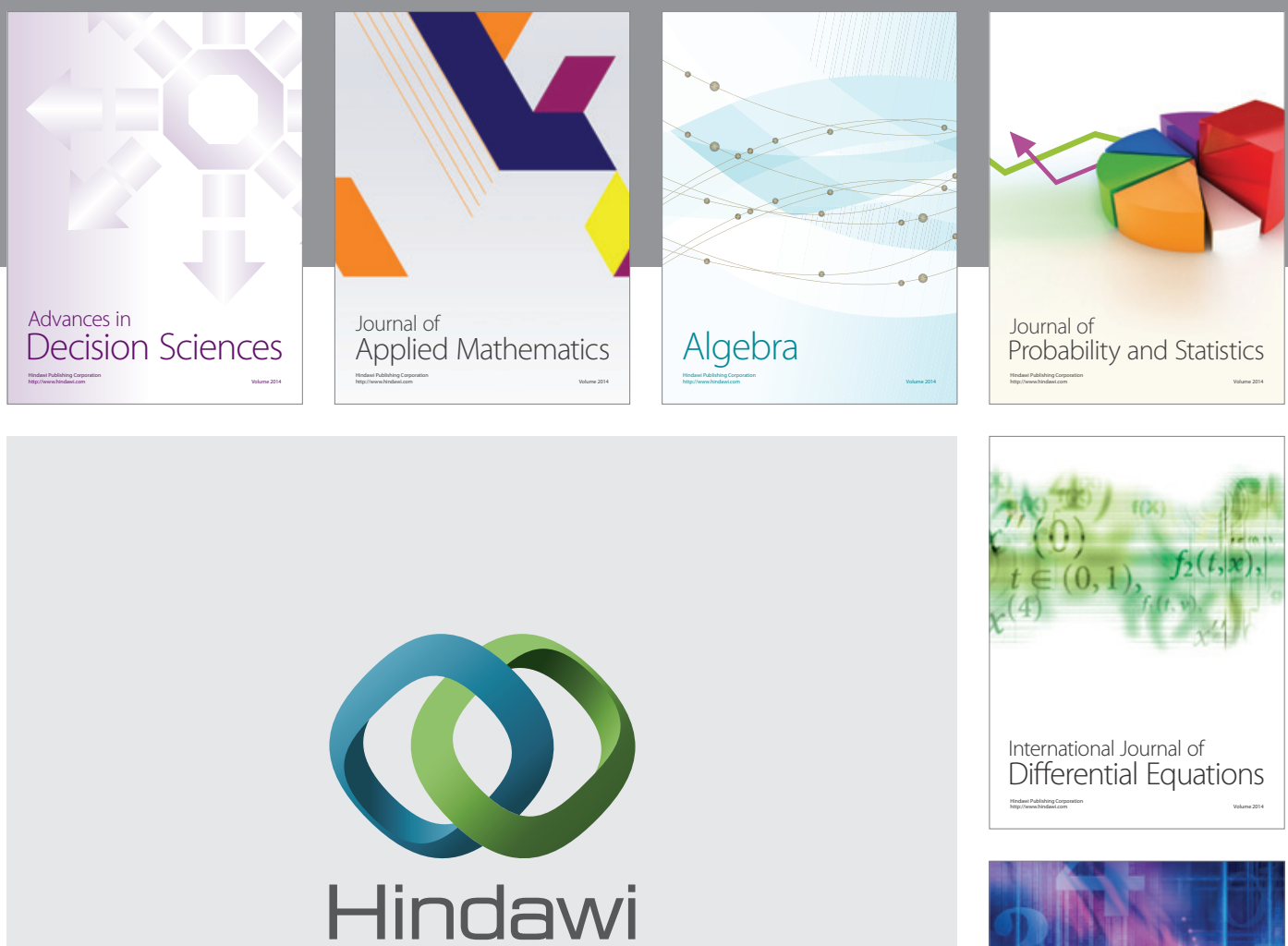

Submit your manuscripts at http://www.hindawi.com
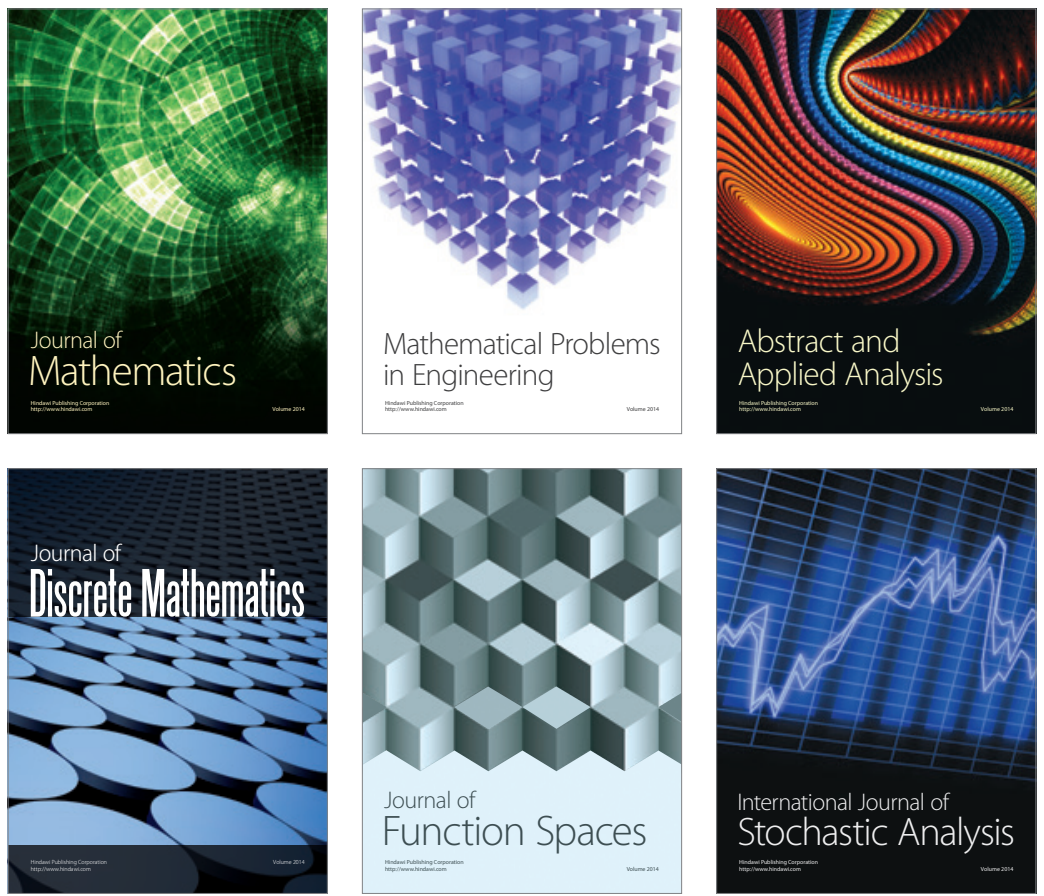

Journal of

Function Spaces

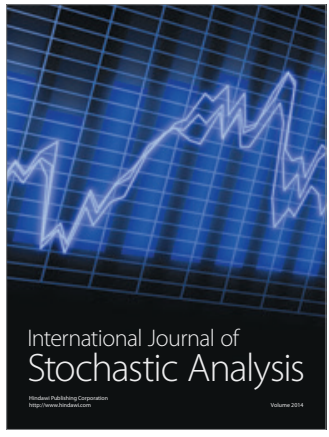

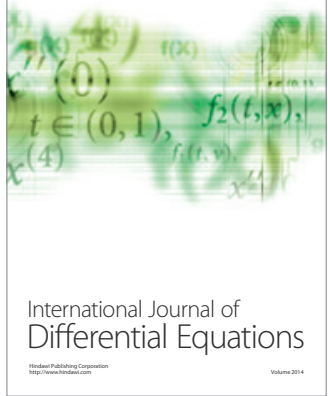
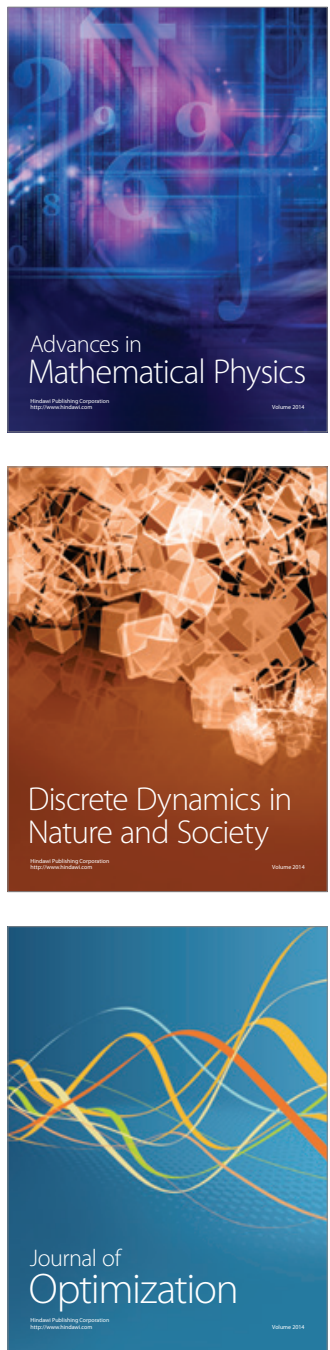